\title{
THE ANCIENT MUSICAL TRADITION IN LATE LATIN WRITERS: $3^{\mathrm{RD}}-5^{\mathrm{TH}}$ CENTURIES \\ Fuensanta Garrido Domené \\ Universidad de Córdoba fgdomene@uco.es Felipe Aguirre Quintero Universidad de las Islas Baleares felipe.aguirre1@estudiant.uib.cat
}

Received: 15 december 2017 Accepted: 25 february 2018

\begin{abstract}
This work is introduction to and a general survey of the treatises written in Latin between the $3^{\text {rd }}$ and $5^{\text {th }}$ centuries that transmitted the ancient Greek musical theory to the Middle Ages. Throughout these pages there will be a concise, eclectic and panoptic view of Latin authors who dedicated their work or part of their work to notions related to the harmonic science of the ancient Greeks. This study will show a "selection" of certain aspects of Greek music theory in its step to the Middle Ages, such as the gradual loss of the vocal and instrumental musical notation, as well as the progressive importance that Rhythmic and Metrics were acquiring into the musical treatises of this era.
\end{abstract}

Keywords: Censorinus, Chalcidius, Macrobius, Martianus Capella, Augustine of Hippo, Ancient Greek Music.

\section{LA TRADICIÓN MUSICAL ANTIGUA EN AUTORES LATINOS TARDÍOS: SIGLOS III-V}

\section{Resumen}

Este trabajo constituye un estudio en el que se hace una presentación y repaso general de los tratados de entre los siglos III y $\mathrm{V}$, en lengua latina, que transmitieron a la Edad Media la antigua teoría musical griega. A lo largo de estas páginas se dará una visión concisa, ecléctica y panóptica de autores latinos que dedicaron su obra o parte de su obra a nociones relacionadas con la ciencia harmónica de los antiguos griegos. En este estudio se evidenciará 
una "selección" de ciertos aspectos de la teoría musical griega en su paso hasta la Edad Media, como la pérdida paulatina de la cuestión notacional vocal e instrumental, así como la progresiva importancia que fue adquiriendo la rítmica y la métrica en los tratados musicales de esta época.

Palabras clave: Censorino, Calcidio, Macrobio, Marciano Capela, Agustín de Hipona, música griega antigua. 


\section{LA TRADICIÓN MUSICAL ANTIGUA EN AUTORES LATINOS TARDÍOS: SIGLOS III-V \\ Fuensanta Garrido Domené \\ Universidad de Córdoba \\ fgdomene@uco.es \\ Felipe Aguirre Quintero \\ Universidad de las Islas Baleares \\ felipe.aguirre1@estudiant.uib.cat}

\section{Introducción ${ }^{1}$}

Una de las grandes joyas que la antigua Grecia ha legado a Occidente es la música. Aunque son muy pocos los tratados musicológicos griegos que han sobrevivido al paso del tiempo y aún menos los documentos que contienen la antigua notación musical, instrumental y/o vocal, la teoría harmónica elaborada por el pueblo heleno fue, es y seguirá siendo un ramo de estudio atractivo.

Gracias a la labor de autores tardolatinos y medievales, la antigua $\mu$ de estudio del individuo latino y medieval. En documentos conservados y fechados en esta época se recoge la teoría musical griega en toda su extensión (teoría harmónica, organológica y notación musical), así como la doble concepción de esta ciencia de acuerdo con las dos escuelas musicales de época antigua: la pitagórica y la aristoxénica. ${ }^{2}$ Debemos a autores como Censorino, Calcidio, Macrobio, Marciano Capela, Agustín, Boecio, Casiodoro o Isidoro de Sevilla la recuperación y compilación de las ciencias de estudio que, poco a poco, fueron conformando el plan de estudio medieval bajo la esfera del Triuium y del Quadriuium. En él, estos autores no solo recuperaron la antigua disciplina harmónica griega con las pertinentes

\footnotetext{
${ }^{1}$ Los autores agradecen a los editores y revisores de Littera Aperta sus sugerencias críticas, que han contribuido a la mejora del presente trabajo.

${ }^{2}$ Eternas rivales, cada una concebía la música desde una perspectiva bien distinta: la pitagórica la entendía en la base del número, estableciendo complejos cálculos en las proporciones de los intervalos musicales, mientras que la aristoxénica, llamada así por Aristóxeno de Tarento, la comprendía en sí, sin necesidad de recurrir a las matemáticas. Destacados pitagóricos fueron Filolao de Crotona, Arquitas de Tarento, Trasilo y el propio Pitágoras (García López et al. 2012: 199-267 y 288-302).
} 
innovaciones y/o variaciones sufridas a lo largo de los siglos, sino que combinaron la música con otras ramas de estudio, como la Gramática, la Retórica o la Poética.

No obstante, dada la envergadura y lo ambicioso de nuestro estudio, dedicaremos este trabajo a los tratados escritos hasta el siglo V. Como es sabido, entre la cuarta y quinta centuria tuvo lugar un acontecimiento que marcó el fin de una época: la caída de Roma en el año 455 y el final del Imperio en 476, cuando Odoacro depuso al último emperador, Rómulo Augusto. Hacia finales del siglo V Teodorico puso fin al gobierno de Odoacro, retomando el aprecio por la cultura y recuperando la admiración por la capacidad administrativa y las tradiciones de la clase senatorial romana. Así, en esta primera parte trataremos los autores que formaron parte de los últimos tiempos del Imperio Romano, ${ }^{3}$ mientras que la segunda la dedicaremos a los cálamos de la nueva época de Teodorico hasta el siglo VII e Isidoro de Sevilla, último paladín enciclopédico de las disciplinas clásicas (Mathiesen 2000: 609-643; Bower 2008: 136-167; Reese 1989: 31-79).

Junto a esta labor de recuperación y transmisión de teoría musical en el mundo latino, no pueden obviarse otros acontecimientos paralelos que también desempeñaron un papel destacado en la supervivencia y difusión de la música griega antigua durante la Antigüedad tardía y el Medievo. Es incuestionable la importancia de la lengua del Islam en la conservación de esta ciencia antigua. Como se sabe, entre los siglos V y XII la lengua del pueblo heleno fue prácticamente olvidada en Occidente y quedó relegada, básicamente, a un ámbito teológico y eclesiástico y no al de la ciencia y el arte. ${ }^{4}$ Asimismo, la expansión del Islam tras la muerte de Mahoma en 632 hizo que varios centros culturales fueran asimilados, poco a poco, a esta nueva cultura. Fue así como a lo largo de la octava y novena centuria muchos tratados griegos de temática científica y técnica y algunos filosóficos (especialmente del corpus platónico y aristotélico) fueron traducidos al árabe o al sirio. De esta manera, los seguidores de Mahoma se convirtieron en los

\footnotetext{
3 Téngase en cuenta que la obra de Marciano Capela fue compuesta, según opinión unánime de la crítica, entre el saqueo de Roma por Alarico (410) y el cruce del Estrecho de Gibraltar por el vándalo Genserico (429) o la toma de Cartago sin lucha por los vándalos (439). A su vez, Agustín murió durante el saqueo y sitio de Hipona por parte de Genserico en el año 430.

${ }^{4}$ Courcelle (1969: 147-148 y 411-421) y Marrou (1985).
} 
garantes y conservadores de las antiguas enseñanzas griegas. ${ }^{5}$ No obstante, y pese a esta tarea de traducción, la intención última del mundo islámico no era sino la de adaptar, transformar y asimilar este legado foráneo al nuevo orden religioso, social e intelectual.

En Occidente, en cambio, el interés que manifestaban los escritores en lengua latina de las primeras centurias hacia estos documentos musicales griegos resultó ser muy diferente, si bien es cierto que, al principio, la atención prestada a las disciplinas matemática y científica quedó reducida a unos pocos nombres y títulos. De esta suerte, la crítica está de acuerdo en que los autores latinos de esta época tenían como tratados canónicos de estas ciencias los escritos de Varrón, Vitruvio y Plinio. ${ }^{6}$ Aunque el conocimiento directo de la ciencia y la filosofía griegas era menos común entre los escritores latinos, hoy se sabe que estos acudían al material matemático y científico griego en obras adecuadas a su tiempo (Mathiesen 2000: 609-614). De hecho, las escuelas latinas contaban tanto con manuales que contenían las disciplinas tradicionales tratadas por Varrón, como con comentarios sobre antiguas obras cuya complejidad llevó a una casi reinterpretación de su contenido bajo la estela de las nuevas corrientes filosóficas o teológicas, como el Neoplatonismo y el Cristianismo.

La teoría musical griega recogida en los escritos de este periodo, en definitiva, permaneció como materia de interés e importancia hasta la caída de Roma, en el año 455, y hasta el final del Imperio Romano de Occidente, en el año 476.

Los autores objeto de análisis en estos trabajos son fiel reflejo de ambos métodos de conservación y transmisión de la antigua ciencia harmónica:

${ }^{5}$ Piénsese, por ejemplo, en la valiosa biblioteca de los Omeyas de Córdoba en el siglo $\mathrm{X}$ que recogía un vasto y variado número de ejemplares del saber antiguo que, más tarde, pasó al resto de Europa gracias a la labor de traducción del árabe al latín.

${ }^{6}$ Los Disciplinarum libri IX, de los que se conservan pocos fragmentos por transmisión indirecta y que se atribuyen a Varrón, ensalzan la formación del hombre libre (de donde "liberal") a través de las nueve artes conocidas y llamadas por ello "Artes Liberales": la Gramática, la Dialéctica, la Retórica, la Geometría, la Aritmética, la Astronomía, la Música, la Medicina y la Arquitectura. El De architectura de Vitruvio da a la música y, en especial, a la harmonía un protagonismo destacado en la construcción de templos, análogos a la del cuerpo humano bien formado. En cuanto a la Naturalis historia de Plinio el Viejo, fue concebida como una especie de enciclopedia que comprendía todo el conocimiento humano del siglo I. 
compendios de la tradición anterior griega y comentarios a determinados autores y/o aspectos relacionados con la harmonía.

\section{Censorino}

Gramático y filósofo romano cuyo akmé suele situarse en el siglo III, de él se conserva una obra Sobre el cumpleaños (De die natali) escrita en el año 238 con ocasión del cuadragésimo noveno cumpleaños de un tal Quinto Cerelio, aunque se sabe por otras fuentes (Cassiod. inst. 2.5.10; Prisc. gramm. 1.16-17, 14.6 y 40-41) que compuso otros tratados sobre gramática y sobre el acento. Como otros escritos de este periodo, el tratado de Censorino se caracteriza por el eclecticismo y simplicidad de sus exposiciones, siguiendo la tendencia de la época, en la que las preocupaciones históricas, científicas y definitorias se reducían a meros catecismos didácticos o a casi anotaciones y compendios técnicos genéricos. ${ }^{7}$ Muestra de ese eclecticismo, reconocido por el propio autor, son los ecos, a lo largo de la obra, de autores tenidos ya por clásicos en el siglo III, tanto del ámbito filosófico (Anaxágoras, Aristóteles, Epicuro, Heráclito, Parménides, Platón o Teofrasto) como del pitagórico (Filolao o Pitágoras) y del científico en sus ramas matemática (Eratóstenes), médica (Hipócrates) y musical (Aristóxeno). Muchas de estas autoridades serán mencionadas y/o aludidas en las secciones doce y trece, de especial interés para este análisis. Sin embargo, a pesar del conocimiento que presumiblemente debió de tener Censorino de los escritos de todos estos personajes, Varrón es, sin lugar a dudas, su fuente principal, una afirmación avalada por la cantidad de veces que lo cita (Sallmann 1983: 21-22). Esta es la razón por la que la crítica tiende a considerar la obra perdida del reatino como la fons et origo de muchos pasajes del De die natali.

Aunque estilísticamente este tratado está escrito como una exposición somera sobre las medidas y los ciclos temporales organizados en días, meses, años, periodos de gestación y edades y duración del hombre, la tradición ha dividido este documento en veinticuatro secciones, de las que solo tres son de nuestro interés. Según Censorino, todas estas medidas y ciclos se manifiestan a través del número, de los movimientos planetarios y del zodíaco.

El primer apartado destacado por su contenido musical es el décimo, en cuyas primeras líneas, con el pretexto de que va a servirse de las reglas de la música para aclarar la dependencia del número en su exposición de los ciclos

\footnotetext{
${ }^{7}$ Como los escritos musicales de Baquio el Viejo o de Gaudencio el Filósofo, autores ubicados temporalmente en este mismo periodo.
} 
y medidas, anuncia que "va a hablar de cosas desconocidas para los propios músicos":

aliqua de musicae regulis huic loco necessaria dicentur, eo quidem magis, quod ea dicam, quae ipsis musicis ignota sunt. (10.1; ed. F. Hultsch, p. 16.17-19)

"Será necesario, para este pasaje, decir algo sobre las reglas de la música, porque, sobre todo, voy a hablar de cosas que los propios músicos desconocen". 8

No obstante, y pese a su intento por ser "original" en el contenido musical de este capítulo y de los que lo siguen, muchas de las definiciones que aquí presenta son comunes a toda la tradición musical anterior, ya sea de tinte pitagórico, ya de tinte aristoxénico. Así las cosas, luego de concebir la música como scientia bene modulandi, Censorino explica de manera muy sucinta, como si de una anotación se tratara, los conceptos de voz (vox, $\varphi \omega v \eta ́)$, sonido

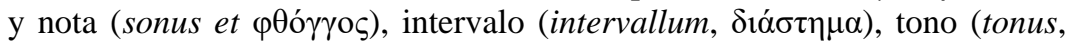

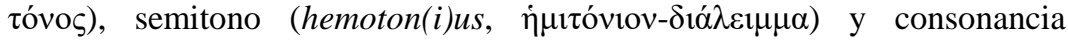

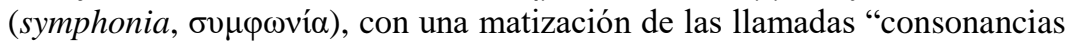

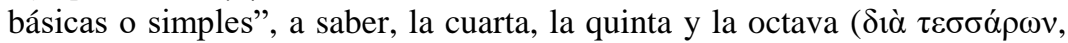

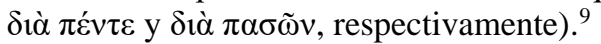

Llegados a este punto, Censorino aprovecha para introducir, como cierre de esta sección, la descripción de los experimentos de Pitágoras con las cuerdas y los pesos para demostrar y diferenciar matemáticamente los intervalos consonantes de los disonantes, una experiencia que luego trasladó a los instrumentos de viento y que respondía a la disposición de los agujeros en el cuerpo del aerófono ( $c f$. Garrido Domené 2012 y la bibliografía allí citada). Su estudio le permitió comprobar que la proporción epítrita

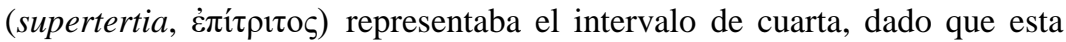
indica la cantidad o el número que contiene la unidad y su tercera parte (4:3); que la proporción hemiólica (sesquialtera, †ं $\mu$ ió $\lambda ı \varsigma$ ) representaba el

\footnotetext{
${ }^{8}$ La traducción de este pasaje y de los restantes son propias.

${ }^{9}$ La doble denominación de semitono responde a su consideración en una u otra escuela: los aristoxénicos emplean ỉ $\delta 1 \alpha ́ \lambda \varepsilon 1 \mu \mu \alpha$ habida cuenta de que para ellos un semitono no es la mitad exacta de un tono, sino que se compone de una parte o semitono mayor ( $\dot{\alpha} \pi \circ \tau$ - $\mu \eta ́)$ y otra parte o semitono menor $(\lambda \varepsilon \tilde{i} \mu \mu \alpha)$ (Theo Sm. 49, Boeth. mus. 3.5 y Michaelides 1978: s.u. "leimma", "apotome" y "hemitonion").
} 
intervalo de quinta al revelar la cantidad o el número que contiene la unidad y la mitad de ella (3:2); y que la proporción epogdoica (superoctava, غ̇ $\pi$ ó $\delta$ oos) representaba el intervalo de octava, pues prueba la cantidad o el número que contiene la unidad y una octava parte de ella (9:8). Estos cálculos de la llamada "harmonía perfecta" son aprovechados por Censorino en la siguiente sección para explicar y justificar por qué el periodo de gestación del individuo debe ser de diecisiete meses, ni más ni menos. La combinación de todos estos conceptos para aclarar, de alguna manera, la diversidad de caracteres y/o cualidades que definen y diferencian a los seres humanos fue un aspecto de la vida tratado ya desde antiguo, como prueban testimonios de Aristóteles (HA 7.3-4) o Arístides Quintiliano (2 y 3.18), en el ámbito de la música, entre otros. Con todo, Censorino revisa en el siguiente capítulo la vinculación de la música con los dioses y con la Humanidad en toda su extensión desde la perspectiva antigua, esto es, cómo la música influye en el hombre en su dualidad cuerpo-alma, una cuestión considerada ya tradicional (por ejemplo, Aristid. Quint. 2).

El capítulo trece es el último que trata la ciencia musical sensu stricto. En él, tomando como fundamento lo explicado en las secciones anteriores, Censorino culmina con la descripción de la música cósmica, es decir, la variedad de sonidos planetarios producida por la diferencia de tamaño, velocidad, trayectoria o posición entre los astros del firmamento. Este aspecto de la teoría musical, conocida como Harmonia Mundi, cuenta con una larga tradición en filósofos y musicólogos anteriores, como en las definiciones ya mencionadas. ${ }^{10}$ En el caso de esta sección, Censorino recoge, de hecho, el modelo de escala planetaria atribuido a Alejandro de Etolia (Godwin 1993: 40-45 y 418-420; Mathiesen 2000: 426-429). De esta suerte, en la construcción de la escala astro-musical los diversos autores que la han tratado identifican cada nota con la posición concreta de un planeta respecto a la Tierra, concebida como centro del sistema.

Como puede verse, Censorino no habla de cosas desconocidas para los propios músicos, como afirmó al comienzo de estas secciones musicales. De hecho, ni siquiera su primera definición en este ramo de estudio, la de música,

\footnotetext{
${ }^{10} \mathrm{El}$ autor a quien debemos la transmisión más antigua de este aspecto de la teoría harmónica es Platón (por ejemplo, en Ti. 34b-36d y 38d; Cra. 397d y 409b-410b; $R$. 616b-617d, sobre la distancia entre los astros; Phdr. 245c-e; o Epin. 986e3-987c7, donde aparecen por primera vez en él los nombres de todos los planetas). La Harmonia Mundi es estudiada en general por Jan (1894) y Luque (2011-2012) y más detalladamente por Garrido Domené (2016: 167-185 y 330-332).
} 
es original, pues la tradición ha conservado definiciones más o menos similares y más o menos elaboradas salidas de cálamos griegos y latinos, como la que ofrecen Arístides Quintiliano (1.4), Baquio el Viejo (Harm. 1, Jan 292.1-2), el texto de Anónimos de Bellermann (\$12 y §29) o el de Excerpta Neapolitana (4-5, Jan 412.17-413.2). De hecho, aunque la expresión musica est scientia bene modulandi parece remontar a Varrón, de nuevo se leerá, aun con variantes, en Agustín, Marciano Capela, Casiodoro e Isidoro de Sevilla, por ejemplo, siendo considerada por algunos críticos modernos como una quasi fórmula que evocaba, por un lado, las antiguas concepciones sobre música desde tiempos pitagóricos, repetidas por musicólogos desde la época helenística hasta el final de la Latinidad, y que se consideró, por otro, como uno de los últimos eslabones entre la tradición latina y la ciencia musical (Aug. mus. 1.2; Mart.Cap. 9.930; Cassiod. inst. 2.5.1; Isid. orig. 3.15).

La tratadística tiende a reconocer una serie de pasajes encabezados con la expresión Fragmentum Censorini como fragmentos conservados de las dos obras atribuidas a este autor por Casiodoro y Prisciano y transmitidos en la tradición manuscrita junto con De die natali hasta que, hacia finales del siglo XVI, Louis Carrion los editó separados. De esta colección de quince fragmentos, solo siete (9-15) son interesantes desde el punto de vista musical. Habida cuenta de que estos pasajes adolecen de componente teórico y de explicaciones más o menos detalladas, la peculiaridad de estos textos radica en lo somero de su exposición y, si acaso, en el empleo casi abusivo de listas nominales y nocionales con las aclaraciones justas. La organización del contenido de los pasajes seleccionados también está falta de una concatenación más o menos lógica, pues su autor pasa de un tema a otro sin justificación aparente retomando lo ya dicho. En este sentido, el fragmento noveno (De musica) consiste en un catálogo de célebres poetas mélicos y músicos de los que añade, en pocas palabras, su logro o logros más destacados. Con el mismo encabezado comienza el fragmento undécimo, en el que Censorino, presumiblemente, ofrece otra definición de música:

Musica est peritia faciendorum et canendorum modorum. eius partes harmonica, organica, rhythmica, crusmatica. (Ps.Cens. frg. 11.1; ed. F. Hultsch, p. 64.11-13)

"La música es la habilidad para hacer y cantar melodías. Sus partes son la relacionada con la harmonía, con los instrumentos, con el ritmo y con la percusión". 
En este y en el siguiente fragmento (De modulatione), por lo que parece, el autor de estos fragmentos enumera y añade breves definiciones de las partes en las que tradicionalmente se divide la música, a saber, harmonica, organica, rhythmica y crusmatica, e introduce las nociones de modo (modus), composición vocal (carmen), tiempo (tempus) y modulación (modulatio), para lo que acude a citas de autoridad, como Aristóxeno de Tarento. La modulación, además, le permite, abordar otras dos cuestiones: los géneros (diatónico, cromático y enarmónico) y las trece tonalidades según el modelo ofrecido por Cleónides, que es, a su vez, el aristoxénico; ${ }^{11}$ y estas, de nuevo, las aprovecha para introducir los nombres de las notas y una versión muy abreviada del desarrollo de las cuerdas de la cítara desde Apolo hasta Terpandro y Timoteo. ${ }^{12}$ Por su parte, los fragmentos décimo (De nomine rhythmi) y del décimo tercero al décimo quinto (De metris id est numeris, De legitimis numeris y De numeris simplicibus, respectivamente) versan sobre cuestiones de rítmica y métrica latinas, acompañadas de escogidos ejemplos. ${ }^{13}$

\section{Calcidio}

Este filósofo cristiano neoplatónico, cuyo floruit suele situarse entre el siglo IV y V, realizó una traducción y comentario del Timeo de Platón, un trabajo que, según la crítica, no disfrutó de mucha influencia a lo largo de la Edad Media quizá por la complejidad de lo que en él se trata. De hecho, parece ser que ni Macrobio ni Isidoro de Sevilla conocieron este texto. Esta obra está dedicada a un tal Osio, tradicionalmente identificado como el obispo de Córdoba o, según Courcelle (1973: 45-53), como un oficial milanés. ${ }^{14}$ Sea como fuere, la labor de traducción y comentario por parte de

${ }^{11}$ Cleonid. Harm. 12 (Jan 203.5-204.15). Cf. Aristid.Quint. 1.10; Ptol. Harm. 2 10; Gaud. Harm. 19 (Jan 347.6-10); Bacch. Harm. 46 (Jan 303.3-6); y Boeth. mus. 1.20. ${ }^{12}$ De los textos antiguos que tratan la cuestión del aumento de las cuerdas de la lira, uid. especialmente Nicom. Exc. 1 (Jan, 266.1-17), 4 (Jan 274.1-275.15) y 6 (Jan 276.8-278.9); Ps.-Plu. Mus. 1141D-1142A; y Boeth. mus. 1.20. De entre las referencias modernas, uid. Sachs (1940: 128-135), Michaelides (1978: s.u. "kithara" y "lyra"), Mathiesen (2000: 258-270), West (1992: 48-70) y Hagel (2009: 76-95 y 133-135).

${ }^{13}$ Sobre los lugares paralelos en estos fragmentos, uid. Sallmann (1983: 71-86) y para un estudio sobre los elementos pitagóricos en la obra de Censorino, uid. Barbera (1980: 257-263).

${ }^{14}$ En su análisis, Courcelle propone que Calcidio fue un cristiano neoplatónico de Milán cuyos escritos llegaron a ser conocidos por Ambrosio. 
Calcidio no abarca el texto original platónico, sino que se limita a la primera mitad, es decir, desde el inicio, 17a, hasta 57c. Llega, por tanto, hasta los pasajes en los que se trata la estructura de los elementos. Su traducción ha sido catalogada como oscura, inexacta y realizada sobre un texto poco correcto.

El trabajo enciclopédico de Calcidio, pues, incluye el diálogo introductorio, en el que Sócrates recuerda una conversación anterior sobre el estado ideal y que concluye con la organización de la que ahora comienza, donde Timeo deberá disertar acerca del cosmos y del hombre y Critias describirá la historia de la Atenas primordial. Así las cosas, Timeo comienza con los principios fundamentales de la primera razón o lógos acerca de la creación, a saber, el ser eterno, perteneciente al ámbito eterno, el devenir, propio del mundo sensible, y lo que lo provoca, el Demiurgo inteligente. Este primer razonamiento sienta las bases de su afirmación de la existencia de un universo como ser viviente dotado de razón, pues así quiso hacerlo el Demiurgo o Maestro Artesano. A partir de aquí, Timeo aborda la descripción de la creación de los seres vivientes eternos, del mundo, del cuerpo del mundo y del alma del mundo. Es en este punto, cuando Timeo está hablando del binomio cuerpo-alma, cuando Calcidio se muestra más fiel a la teoría musical platónica y al alma del mundo. Este extenso pasaje lo ocupan los capítulos 40-55 (Wrobel 106.4-123.5) de la traducción de Calcidio, correspondientes a 36a-37a del original platónico.

La teoría musical tratada en estas páginas, de evidente corte pitagórico, se reduce a la explicación de la harmonía a partir de las proporciones doble y triple descritas por Platón y representadas según la fórmula 6:8:9:12, un cálculo que contiene la media harmónica, aritmética y geométrica, herrajes de la estructura geométrico-musical del mundo. ${ }^{15} \mathrm{La}$ inclusión, asimismo, de la historia del descubrimiento, por parte de Pitágoras, de los números consonantes a partir del experimento con pesos suspendidos de cuerdas sirve de puente para abordar la cuestión de las caracterizaciones numéricas de las consonancias simples y compuestas, del tono y del llamado leimma (Heath 1981: 85-90; Mathiesen 1998: 19-23 y 2000: 399-401; Garrido Domené 2012 y 2013). Además de los intervalos o consonancias base (cuarta, quinta y octava), los griegos reconocieron como consonantes otros intervalos

${ }^{15}$ En este punto, Calcidio se detiene en la imagen de Platón de los dos círculos giratorios del cosmos y de la dualidad de la Esencia -la Indivisible y la Divisible- y su naturaleza -lo Mismo y lo Otro. $C f$. Aristid.Quint. 3.24, Godwin (1993: 60-63 y 426) y Mathiesen (2000: 574-576). 


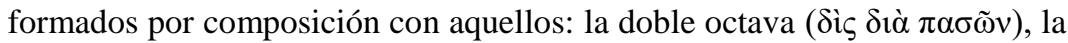

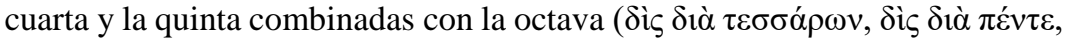

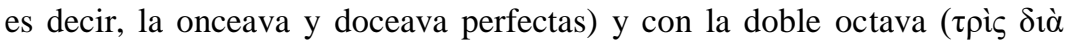

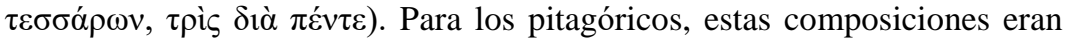
consonantes porque su expresión numérica resultaba de las proporciones más simples: la octava (2:1), la quinta (3:2), la cuarta (4:3), la doceava (octava y quinta, $3: 1$ ), la doble octava (4:1) y la onceava (octava y cuarta, $8: 3){ }^{16}$

La "dificultad" de Calcidio estaba en interpretar del Alma del Mundo, un concepto "pagano", en términos cristianos. Para ello, buscó conexiones entre la concepción que ambas corrientes tenían acerca de la naturaleza del ser humano. Es en este contexto cuando Calcidio manifiesta la importancia de la música:

Procul dubio musica exornat animam rationabiliter ad antiquam naturam reuocans et efficiens talem demum, qualem initio deus opifex eam fecerat. (Chalc. comm. 267; Wrobel 298.22-24) ${ }^{17}$

"Sin lugar a dudas, la música embellece racionalmente el alma, volviéndola de nuevo en su antigua naturaleza y haciéndola exactamente como Dios, el creador, la había creado al principio".

\section{Macrobio}

Natural de alguna de las provincias más latinizadas del Imperio, este adalid de cálamo pagano y conocido como Macrobio Ambrosio Teodosio es un personaje del que se tienen pocas noticias. Tanto es así, que su identificación ha fluctuado entre un prefecto en Hispania (399-400), un procónsul en África (410), citado en el códice Teodosio, o un prefecto pretoriano en Italia (430). A día de hoy, esta última propuesta es la más aceptada (Cameron 1966; Navarro Antolín 2006: 7-26 y la bibliografía allí citada).

Pese a los pocos datos que de él se tienen, se sabe que escribió tres obras, conservadas total o parcialmente: Comentario al Sueño de Escipión (In somnium Scipionis commentarium), Saturnales (Saturnalia) y Sobre las diferencias y semejanzas del verbo griego y latino (De differentiis et societatibus Graeci Latinique uerbi). Mientras que las dos primeras están dedicadas a su hijo Fl. Macrobio Plotino Eustacio, la última, de la que solo quedan unos cuatro fragmentos, consiste en un tratado en el que se cotejan

16 Vid. Michaelides (1978: s.u. "symphonia, symphonos").

${ }^{17}$ Cf. Pl. Ti. 47d. 
los sistemas verbales griego y latino para cada uno de los siete accidentes gramaticales que presentan (Keil 1961: 595-655). Sea como fuere, solo la primera es de interés para el presente trabajo, pues cuenta con un contenido musical de tendencia pitagórica muy considerable (Barbera 1980: 276-281).

Como se sabe, el Sueño de Escipión cerraba el Sobre la república (De re publica) ciceroniano (6.9-29), la única parte de este escrito conocido en el Medievo y que el famoso arpinense tomó, a su vez, del Mito de Er de Platón, pasaje que pone fin a su Respublica. En esas páginas, Cicerón cuenta la aparición, en un sueño, de Escipión Africano el Viejo a Escipión Emiliano, su nieto adoptivo, para contarle su propio destino y el de su país, para revelarle la gloria de la virtud tras la muerte y para intentar hacerle ver cómo es el cosmos y qué lugar ocupan la Tierra y el hombre dentro de él. Grosso modo, el comentario de Macrobio se centra en rasgos destacados del escrito de Cicerón, como su lenguaje dramático, la imagen de la harmonía de las esferas y las consideraciones sobre la naturaleza y el ascenso del alma. Así las cosas, Macrobio no hace un comentario sensu stricto del texto ciceroniano, sino que, bajo el velo del Neoplatonismo, presenta una serie de teorías sobre los sueños, sobre las propiedades místicas de los números, sobre la naturaleza del alma, sobre astronomía y sobre música, todo ello avalado por citas de autoridad que muy probablemente no leyó en su totalidad. De ellas, sus fuentes principales son Plotino y Porfirio, si bien Virgilio también es mencionado aunque con intención estética. Pese a su estilo catalogado como "desigual" por no unificar estilísticamente las fuentes empleadas, la riqueza de este documento está en la conservación directa y su consecuente transmisión de ideas y conceptos neoplatónicos no conservados en ningún otro lugar (Navarro Antolín 2006: 26-113, con bibliografía).

En cuanto a los aspectos destacados, unos se presentan de manera clara en el libro primero y se continúan, veladamente, a lo largo del escrito, mientras que otros se anuncian o intuyen al comienzo y se desarrollan más ampliamente en el libro segundo. El material, por tanto, tratado por Macrobio y que es de nuestro interés se refiere a la clasificación de los sueños, al descubrimiento, por parte de Pitágoras, de las consonancias musicales, a la teoría pitagórica del número, a los movimientos de las esferas celestes y planetarias, así como a sus sonidos harmónicos, a la naturaleza de la virtud, a la diferenciación entre mortalidad e inmortalidad, a las hipóstasis neoplatónicas -el Uno, el Intelecto o Mente del mundo (nous) y el Alma- y la superioridad de Platón sobre Aristóteles en lo que a su visión del alma se refiere. La poikilía de disciplinas diversas habla por sí misma: consideraciones filosóficas sensu stricto (passim), pasajes sobre aritmética 
(1.5-6), astronomía (1.14.21-1.22.13), música (II 1-4) y geografía (2.5-9). Semejante manejo de conocimiento propició la inclusión de Macrobio, por parte de diversos autores (por ejemplo, Flamant 1997: 305-306; Scarpa 1981: 21ss.; Regali 1990: 8-9), en la corriente enciclopedista que caracterizó la Antigüedad Tardía de los siglos IV-VI y que volverá a aparecer en autores como Marciano Capela, Casiodoro, Boecio o Isidoro de Sevilla, transmisores, de una manera más accesible, de las artes liberales y de las enseñanzas filosóficas de época clásica.

Entre tales cuestiones, por tanto, hay algunas eminentemente musicales de tradición pitagórica a las que, como se ha visto, Macrobio dedica las cuatro primeras secciones del libro segundo. Su "Tratado de música", en la primera sección de este libro, comienza con la Harmonía de las Esferas, haciendo del aire golpeado el principio básico por el que se emite un sonido, que será harmónico o no dependiendo de la ley numérica que lo precise. Esta primera conclusión acerca de la creación de sonido, en general, y del planetario, en particular, la concreta con la ya tradicional historia del descubrimiento de Pitágoras de las reglas matemáticas que rigen las consonancias, las relaciones entre ellas y, por tanto, la harmonía. La segunda sección continúa lo tratado en la anterior acerca de la música planetaria, si bien este apartado está más centrado en la explicación platónica de las relaciones harmónicas y el Alma del Mundo descritos por el filósofo de Atenas en su diálogo Timeo. Partiendo, por tanto, de la diferenciación entre los cuerpos sólidos y la diversidad de cuerpos matemáticos, Macrobio comenta lo dicho por Platón en Ti. 35b-36a, un pasaje, podría decirse, casi de obligada cita e interpretación en los autores de corriente neoplatónica de esta época. En su exégesis acude, como es de esperar, a un pasaje de la traducción latina que Cicerón hizo de este texto (rep. 6.18).

Continuando con la prueba de esa harmonía celeste, la tercera sección está dedicada a dicha demostración, para lo que Macrobio acude a las interpretaciones alegóricas. Según él, filósofos y teólogos han dado fe de la existencia de esta música celestial recurriendo a personajes míticos (sirenas y musas), a ritos religiosos, donde la música vocal e instrumental jugaba un importantísimo papel en el sacrificio, o a mitos musicales protagonizados por músicos legendarios, como Orfeo y Anfión. ${ }^{18}$ Así las cosas, y habiendo quedado demostrada la existencia de la música celestial, Macrobio pone fin

18 Sobre las referencias de otros autores que también trataron la música en las ceremonias religiosas, uid. S.E. M. 18; Cens. 1.2.2.; Ps.-Plu. Mus. 1140D-E; Aristid.Quint. 2.4; e Isid. orig. 3.16.2-3. 
a esta sección explicando cómo surgen los intervalos planetarios a partir de las distancias dadas entre los astros, un cálculo que, según nuestro autor, remonta a Arquímedes pero que fue rechazado y "corregido" por el platonismo al no respetar las progresiones de los números dos y tres ( $c f$. Navarro Antolín 2006: 343-344 con nn. 70 y 72; Godwin 1993: 64-70 y 426427). Una vez establecido el cómputo adecuado, la sección cuarta pone fin al "Tratado de música" con una concreción acerca de la harmonía que producen las esferas planetarias, diferenciando, para ello, la altura de los sonidos celestes, esto es, su gravedad o agudeza. Esta observación y comentario, con sus errores y aciertos, permite a Macrobio organizar los sonidos planetarios en siete, volviendo, así, a la mística del número que trató en el primer libro (1.6.34). Esta cifra, de hecho, sagrada y venerada por y desde los primeros pitagóricos, tenía en sí una fuerte evocación simbólica: cada uno de los siete astros produce un sonido, correspondiente a cada una de las siete vocales que forman parte del alfabeto griego $(\alpha, \varepsilon, \eta, 1, o, \omega, v)$ y, de ahí, de los siete sonidos principales. Esto, a su vez, suscita determinadas correspondencias, además de la ya indicada: las siete edades del hombre, los días de la semana, los tonos de la escala primitiva, el número de los planetas caldeos, los siete sabios, las siete puertas de Tebas o, incluso, el nacimiento de Apolo el séptimo día del mes Bysios. ${ }^{19}$ Estas matizaciones, no obstante, sirven para aclarar -dice Macrobio- lo dificultoso de las palabras de Cicerón sobre música. De cualquier manera, nuestro autor cierra su tratamiento sobre esta disciplina dedicando unas breves palabras a los genera harmoniae enarmónico, diatónico y cromático- y a la imposibilidad humana de escuchar esta música celestial ( $c f$. Garrido Domené 2016: 167-185, especialmente 184-185).

La fortuna de este autor y de Calcidio a lo largo de la Edad Media fue muy considerable, si bien este parece haber ejercido un influjo más directo en la teoría musical del latín tardío y medieval, llegando a ser citado, incluso, por autores muy posteriores, como Bernelino, Engelbert von Admont, Jacques de Liège y Franchino Gaffurio (cf. Phillips 1990; Huglo 1990).

\section{Agustín de Hipona}

Agustín (354-430), Obispo de Hipona, fue un polígrafo que manifestó una inquietud por mantener viva la herencia clásica a través de la

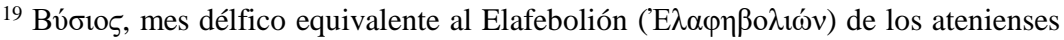
(segunda mitad de marzo y primera de abril).
} 
conservación y transmisión de las disciplinas varronianas, como luego se verá en el caso de Marciano Capela. Sin embargo, esta primera preocupación "de juventud", por así decir, fue evolucionando como evolucionó su propia vida, de manera que en la redacción de su obra sobre música, más que conservar y transmitir una tradición anterior, se dedicó a reflejar el importante papel que la música pudo desempeñar en el contexto cristiano adaptado al Neoplatonismo. Aunque el propio Agustín manifestó su intención de escribir un tratado sobre cada una de ellas (retract. 1.6 y epist. 101.3), hoy se sabe que solo dedicó trabajos especializados a la gramática y a la música y, de ellas, solo el tratado Seis libros sobre música (De musica libri sex) se ha conservado, un texto que aborda, grosso modo, la métrica del lenguaje ( $c f$. Luque y López 2007: 7-81). En este sentido, la crítica ve en este texto de Agustín una complementación de los tratados sobre el ritmo y la métrica de Hefestión y de las secciones de la obra de Arístides Quintiliano que abordan esta temática. Con todo, existe común acuerdo en ver una destacada influencia de Porfirio, cuya obra nuestro autor pudo conocer en traducción (Theiler 1933).

Con un programa original en seis libros dedicados al ritmo y otros seis dedicados a la melodía, el De musica agustiniano vio reducida su exposición a cinco libros sobre cuestiones relacionadas con el ritmo, escritos, presumiblemente, hacia el año 387 -más o menos la misma época en la que la crítica está de acuerdo en situar la culminación de su tratado Sobre gramática (De grammatica)-, y a los que seguía un sexto libro sobre metafísica musical, como lo ha calificado parte de la crítica, o sobre el componente filosófico-teológico de la música, según otros. En él, la actitud de Agustín recuerda la de neoplatónicos anteriores, pues otorga una atención especial al número y a la proporción, las herramientas o los medios para entender el plan harmónico del Creador (aquel Artesano o Demiurgo) en el universo. De hecho, pasó casi un lustro desde la redacción de la parte dedicada al ritmo y esta última, siendo evidente, por tanto, un cambio de proceder en el autor justificado por Thomas J. Mathiesen (2000: 619-620) por su conversión y extraordinaria combinación de Neoplatonismo y Cristianismo. ${ }^{20}$ Sea como fuere, lo más característico de este documento es,

${ }^{20}$ Con todo, los manuscritos tienden a presentar este libro sexto independiente de los primeros. Cf. Ortega (1988: 50-69), Huglo (1990: 170), Luque y López (2007: 2838), Prada (2014: 11-59) y Jacobsson (2017: 1-62). Asimismo, la visión de la música, por parte de Agustín, como el instrumento de alabanza a Dios y de la elevación del 
sin lugar a dudas, la elevación de la música como ciencia divina, gracias a la cual el alma podía purificarse con la numerositas, esto es, la propiedad de la música en forma de relación numérica celestial.

Que los cinco primeros libros se ajustan a un formato de presentación que remonta a Baquio el Viejo es un hecho más que evidente e incuestionable por parte de la crítica. Dicho método, si acaso uno de los más empleados en los tratados de teoría musical latina de la época, consiste en una especie de diálogo entre maestro y discípulo, lo que otorga a estos documentos cierto carácter de simplicidad. No obstante, en el caso de Agustín esa simplicidad es aparente, pues las cuestiones que aborda en su tratado musical -sobre el metro y la versificación- requieren de unos conocimientos previos no exiguos. Interesado, como Bacon, en el metro y en el acento, los cinco primeros libros muestran un carácter técnico. En el primero de ellos ofrece una concepción de música vinculada, veladamente, a otras disciplinas, haciendo especial hincapié en la enorme importancia que esta alcanzó incluso más que la gramática- en el conocimiento y comprensión del sonido del lenguaje. ${ }^{21}$ Para ello, además, incluye ciertas nociones aritméticas, desarrollando los principios básicos de proporción según la tradición pitagórica ( $c f$. Otaola 2005). Así las cosas, para Agustín en el número está el verdadero conocimiento de la música, un conocimiento, según Thomas J. Mathiesen (2000: 620, n. 38), desconocido para los cantores theatrici, a los que se les habría supuesto expertos en música (1.6). En esta misma línea se muestra en lo que resta del primer libro, ensalzando la importancia del número en términos harmónicos para resaltar, así, las proporciones del tiempo expresadas en el ritmo y en el metro:

Tempus est autem ad illos motus redire tractandos et discutiendos, qui huic disciplinae proprie tribuuntur, et propter quos ista de numeris, de alia scilicet disciplina, quantum pro negotio satis uisum est, considerauimus. (Aug. mus. 1.27)

"Es momento, no obstante, de volver a tratar y a examinar aquellos movimientos que se atribuyen, propiamente, a esta disciplina, y por los cuales hemos considerado lo relacionado con los números, obviamente

alma hasta lo infinito y lo inexpresable vincula este tratado con las Enarrationes in Psalmos y otras colecciones (Cattin 1987: 161).

${ }^{21} C f$. Luque (2014). Su definición de música como scientia bene modulandi (1.2) y scienctia bene mouendi (1.3) evidencia su conexión, de hecho, más con la tradición musical griega que con la retórica. 
perteneciente a otra disciplina, cuanto ha parecido suficiente según la tarea".

Los libros II-V versan, de manera general, sobre el ritmo, entendido como la articulación e intensidad, incluso, de la duración de los sonidos. No obstante, se echa en falta en estos libros el otro componente del sonido musical, la harmonía, con todos sus ramos de estudio (altura y articulación tonal, intervalos, escalas, modos...). Este modelo agustiniano de música "humana", en lenguaje boeciano, en tres grupos (harmonica, rhythmica, metrica) será empleado, posteriormente, por Casiodoro. Sea como fuere, una detallada lectura de cada uno de estos libros concreta su contenido. El libro segundo, pues, se centra en el verso, en las sílabas y en los pies rítmicos. En sus páginas, Agustín hace manifiesto el tratamiento que gramáticos y músicos otorgan al verso: mientras que estos emplean la razón y el sentido, aquellos recurren a la autoridad de la gramática. Por ello, una vez más nuestro autor ensalza la primacía de la música en la definición del ritmo y del metro del verso (Mathiesen 2000: 40-41). Estos tres elementos -ritmo, metro y verso- no son exactamente lo mismo, por lo que es menester, dice Agustín, establecer sus diferencias y no caer en considerarlos como sinónimos. A este empeño dedica el tercer libro, aportando numerosos ejemplos sacados de todos los rincones de la literatura clásica. El libro cuarto continúa lo iniciado en el anterior, si bien en este su tratamiento de los metros es mucho más detenido y atento, proponiendo, incluso, posibles combinaciones en verso, de lo que se ocupa en el quinto libro.

Con un contenido y un tono claramente neoplatónico, el último libro muestra evidencias de que Agustín debió de conocer, al menos a través de la traducción de Cicerón, pues no sabía griego, el Timeo platónico. ${ }^{22}$ En este libro que cierra su escrito sobre música, luego de aclarar su sentido en el conjunto de la obra y de justificar los libros anteriores (6.1.1), lleva el número y la proporción a un plano no corpóreo, donde permanecen en la memoria y donde son eternos. Este es el componente filosófico-teológico de la música anteriormente aludido. Así las cosas, pueden establecerse dos partes en el contenido de este libro: una dedicada a la percepción del ritmo, es decir, a los llamados "números del alma" y sus clases (6.2.2-8.22), y otra centrada en el placer estético, en la contemplación filosófica, donde se consideran los "números eternos", que proceden de Dios (6.9.23-17.58). Se destacan, por tanto, al comienzo de este libro los cinco genera de números en virtud de su

${ }^{22}$ Así lo manifiesta en conf. 1.13.20 y en c.acad. 3.4.7 (Courcelle 1969: 149-196). 
ámbito de "aplicación": sonus, sensus audientis, actus pronuntiatis, memoria y iudicium. A continuación, tras revisar y considerar las virtudes (prudentia, temperantia, fortitudo, iustitia), determina la dimensión sensorial de la música relacionada con los principios numéricos de la proporción, una cualidad esta capaz de llevar al alma al amor a Dios (Mathiesen 2000: 621 y n. 43).

Agustín es, en definitiva, "un primer paso para ilustrar al mundo cristiano la teoría que ningún docto debería ignorar y la premisa para convencer de que teoría y práctica podían y debían (aunque el proceso se prolongó muchos siglos) marchar juntas" (Cattin 1987: 82).

\section{Marciano Capela}

Contemporáneo de Agustín, Marciano Min(n)e(y)o Félix Capela, oriundo de Cartago, es el autor del que podría considerarse el tratamiento más completo de las nueve disciplinas varronianas, de las que, si bien su primera intención fue presumiblemente presentarlas tal cual, acabó tratando siete de aquellas nueve tras "eliminar" la Medicina y la Arquitectura. Ubicado temporalmente entre finales del siglo IV y comienzos del V, ${ }^{23}$ su Sobre las nupcias de Filología y Mercurio (De nuptiis Philologiae et Mercurii) fue tenida como una de las obras más importantes de la Edad Media, reconocida, incluso, como herramienta esencial para la formación del individuo por compendiar y conservar el material básico de las disciplinas clásicas ( $c f$. Navarro Antolín 2016: XV-CXIII y la bibliografía allí citada).

El enciclopedismo, por tanto, es uno de los rasgos que definen este escrito, un aspecto propio de la época y reflejado en el esfuerzo que este autor y otros anteriores llevaron a cabo para salvaguardar y transmitir al mundo medieval el conocimiento de las artes liberales y de la filosofía y la ciencia clásicas. Pese a ello, y como correlato, la labor compiladora de estos hombres de letras sacrificó la precisión de los avances y logros de la mente clásica, pues no son pocos los casos de comentaristas y compiladores intermedios que separaron al compilador del original griego o latino. Con todo, aunque por estas recopilaciones se perdieron originales, al menos se conservó la transmisión de sus contenidos.

\footnotetext{
${ }^{23}$ Esta ubicación temporal, con la que la crítica está de acuerdo de manera unánime, lo hace contemporáneo no solo de Agustín, sino de Símaco, Servio, Macrobio y Rutilio Namaciano. Vid. n. 2 y Stahl et al. (1971: 9-20), Shanzer (1986: 1-28), Cristante (1987: 27-31), Ramelli (2001: VII), Guillaumin (2003: VII-XVI) y Navarro Antolín (2016: XV-XXI).
} 
Junto a este enciclopedismo, el texto de Capela destaca por su técnica literaria, en la que hace gala del empleo del prosimetron, con la combinación de verso y prosa en la senda de la antigua sátira menipea de Varrón, Menippo de Gádara o la Apocolocynthosis de Séneca. ${ }^{24}$ El estilo hermenéutico, además, ha sido reconocido por la crítica como otra de sus peculiaridades, así como el gusto por el elemento alegórico, tan propio de la época.

De nuptiis Philologiae et Mercurii narra en nueve libros cómo Mercurio, cansado de su soltería, decide casarse. Escrito en forma de sátira, ${ }^{25}$ que el propio autor llama fabula, ${ }^{26}$ Capela describe a su hijo, en los dos primeros libros, las bodas místicas de Mercurio y Filología, una doncella propuesta por Apolo y que finalmente fue elegida por aquel tras rechazar a Sofía, Mántica y Psiqué. La condición mortal de la prometida debe ser eliminada para poder ser admitida en el mundo divino, así como su unión con Mercurio. Para ello, se convoca una asamblea divina ante la cual la novia ha de presentarse, ya inmortalizada, acompañada de un séquito de siete doncellas, la dote que Febe, en nombre de Apolo, le regala y que resultan ser las Artes Liberales: Gramática, Dialéctica, Retórica (lo que en la Edad Media se conocerá como Triuium), Geometría, Aritmética, Astronomía y Harmonía (el Quadriuium medieval). En el libro segundo se describen los preparativos de Filología para su apoteosis y enlace con Mercurio. Ayudada por Frónesis, su madre, la novia es acompañada en todo momento por las nueve Musas, que entonan, cada una, un canto de alabanza a la futura esposa, las cuatro Virtudes, Filosofía y las tres Gracias. Tras purificar su parte mortal al beber

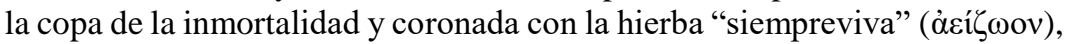
asciende, en la litera nupcial, al palacio de Júpiter. En su camino a través de

\footnotetext{
${ }^{24}$ Navarro Antolín 2016: XXVI, n. 33 presenta amplia bibliografía que defiende la composición de la obra de Capela siguiendo el modelo estilístico de las sátiras menipeas

${ }^{25} C f$. nota anterior. Gaspar Scioppius, a comienzos del siglo XVII, afirma que obras "serias" como Las nupcias de Capela o La consolación de Boecio pueden considerarse satyrica por la variedad de sus temas y por el uso del prosimetrum: recte satyricon appellatur opus, in quo quasi per Saturam uaria argumenti uario ac multiplici stili genere pertractantur, cuiusmodi sunt Petronii Arbitri, Martiani Capellae et Boethii de Consolatione Philosophiae libelli... (Satyricon, fol. 271r; "es llamada correctamente satírica la obra en la que se expone, como en desorden, diversidad en la variedad de argumento y en la múltiple forma de estilo, como son los opúsculos de Petronio Árbitro, de Marciano Capela, el Sobre la consolación de Filosofía de Boecio...").

${ }^{26}$ Propiamente "relato fantástico", "narración mitológica" o "cuento alegórico".
} 
las esferas, junto con Juno Prónuba, su comitiva de Musas y otras asistentes, se encuentra con todo tipo de personajes y criaturas que habitan las diversas regiones del universo hasta llegar a la esfera de las estrellas fijas. ${ }^{27}$ Allí, y antes de presentarse ante Júpiter y ante la asamblea divina, formula una serie de plegarias silenciosas para, ya en la Vía Láctea, concluir los esponsales sacros. Es entonces, a partir del tercer libro, cuando Capela presenta y trata cada Arte, pues una a una ha de explicar su saber ante el consejo convocado por Júpiter: Gramática (III), Dialéctica (IV), Retórica (V), Geometría (VI), Aritmética (VII), Astronomía (VIII) y Harmonía (IX).

Comienza el último libro del tratado con una queja, por parte de Venus, por lo extenso de las exposiciones de las compañeras de Filología. Habida cuenta de que aún están esperando para hacer su disertación Harmonía, Medicina, Arquitectura y las artes proféticas, y ante la advertencia de Luna de que pronto tendrá que dejar la asamblea para hacer su ronda por el cielo, los dioses acuerdan que con Harmonía concluirá el consejo. ${ }^{28}$

Descrita como una figura sublime $(\$ 909-\S 910)$, y tras resaltar los beneficios de la música y, especialmente, su ancestral influjo en el comportamiento de hombres y fieras (\$921-§929), Harmonía refiere en su discurso los principios de la harmónica, de la rítmica y de la métrica, para lo que Capela parece basarse en Arístides Quintiliano pese a no mencionarlo. ${ }^{29}$ La crítica está de acuerdo en reconocer a Arístides como la fuente principal para este libro, del que Capela obvia aquel material que rompe la estructura narrativa de su obra, como las cuestiones notacionales (Aristid.Quint. 1.9 y 11). ${ }^{30}$

Previo a su discurso propiamente dicho, Harmonía considera conveniente aclarar ciertos aspectos fundamentales que facilitarán - dice- la comprensión de su ulterior explicación. Así, a lo largo de cinco epígrafes (§930-§935) Capela describe someramente, en boca de Harmonía, los conceptos de tono,

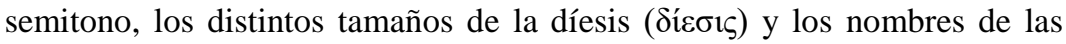

${ }^{27}$ La narración de este pasaje deriva, muy probablemente, de Plin. $H N$ 2.84. $C f$. Godwin (1987: 126) y Schueller (1988: 261).

${ }^{28}$ Medicina y Arquitectura, pese a estar presentes, han de guardar silencio por tratar asuntos mortales y mundanos, tal y como propio Capela explica en $\$ 891$.

${ }^{29}$ Sí menciona, en cambio, a Varrón, de quien parece tomar los beneficios y poder de la música. Sobre los préstamos de Arístides Quintiliano tomados por Capela, uid. Deiters (1881) y Stahl et al. (1977: 53-54).

${ }^{30}$ Navarro Antolín (2016: XXXIX-XLI) estudia las fuentes de los llamados libros enciclopédicos. 
notas, ${ }^{31}$ deteniéndose en la explicación de altura y de los tres intervalos consonantes básicos y culminando con los 15 tropoi. Una vez nombrada la división tripartita de la música según Laso de Hermíone -la materia (ì $\lambda$ ıóv),

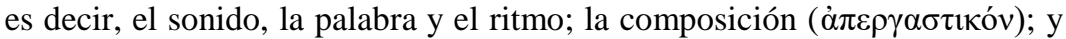
la ejecución ( $\dot{\varepsilon} \xi \alpha \gamma \gamma \varepsilon \lambda \tau \imath \kappa o ́ v)$, en la que se cuenta la instrumentación, el canto

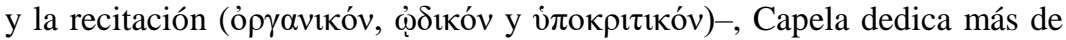
medio centenar de epígrafes a las partes canónicas de la ciencia harmónica (\$938-\$966: nota, intervalo, escala, género, tono, modulación y melopeya) y a los ritmos y la ritmopeya ( $\$ 967-\S 995)$. En este vasto discurso, además de los ecos a Arístides Quintiliano que ya se han mencionado, hay casos en los que Capela parece remitir a la Introductio harmonica de Cleónides, un músico seguidor de la doctrina de Aristóxeno, o a la obra rítmica del propio Aristóxeno ( $c f$. Mathiesen 2000: 627-628). Sea como fuere, cuando Harmonía concluye su discurso, Capela pone fin a su tratado no sin antes recomendar a su hijo su lectura $(\$ 997-\S 1000)$. Esta recomendación se hará extensiva, aun de manera tácita, como texto base en los programas de formación de la época.

\section{Conclusión}

Hemos intentado presentar en estas páginas una visión de conjunto de los tratados que entre los siglos III y V conservaron parte de la antigua teoría musical griega. En nuestro repaso, sin entrar en cuestiones eminentemente técnicas (la clasificación de las harmonías, la oscilación del semitono en el sistema o sistemas tetracordales o el cómputo de los intervalos y microintervalos según las distintas escuelas musicales tradicionales, por ejemplo), hemos intentado destacar aquellos documentos más representativos en cuanto a época y contenido, ofreciendo una panorámica general de sus autores y de las inquietudes que los llevaron a componer estos escritos. La evolución en el tratamiento de la música teórica a lo largo de estas obras evidencia, además, la propia evolución en el pensamiento filosófico-teológico de la época, así como los gustos compositivos y estilísticos. Estas nociones y avances en la cultura teórico-musical se

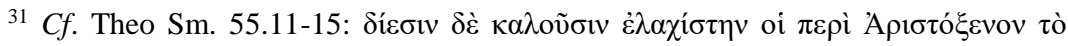

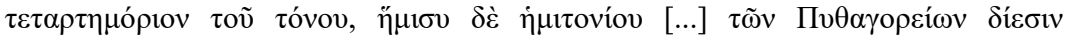

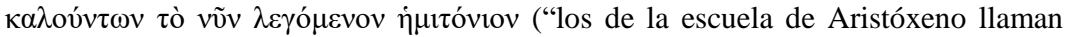
díesis al intervalo más pequeño, el de cuarto de tono, la mitad de un semitono, [...] mientras que los pitagóricos llamaban díesis al ahora llamado semitono"). 
complementarán con la segunda parte de nuestro análisis. De cualquier manera, el importantísimo papel en la transmisión de la cultura clásica al Medievo de los autores aquí presentados, y los que se analizarán en la segunda parte de este trabajo, es innegable. Su reconocimiento actual como garantes de la cultura antigua es, sin duda, más que merecido. 


\section{Obras citadas}

Barbera, André (1980). The Persistence of Pythagorean Mathematics in Ancient Musical Thought. Ph. D. Dissertation. Chapel Hill, NC: University of North Carolina at Chapel Hill.

Bower, Calvin M. (2008). "The transmission of ancient music theory in to the Middle Ages". En Thomas Christensen (ed.). The Cambridge History of Western Music Theory. Cambridge: Cambridge University Press.

Cameron, Averil (1966). "The Date and Identity of Macrobius". Journal of Roman Studies 56: 25-38.

Carrion, Louis (ed.) (1583). Censorinus de die natali. Noua editio Lud. Carrione recensente, augente et pristino ordini suo restituente. Eiusdem argumenti fragmentum incerti scriptoris antea cum eodem Cesorini de due natali libro continenter impressum, nunc uero ab eodem Lud. Carrione separatum, correctiusque et capitibus aliquot ex ueteri libro additis auctius editum. Lutetiae: Aegidius Beysius.

Cattin, Giulio (1987). Historia de la música, 2. El Medievo. Primera parte. Madrid: Turner Música.

Courcelle, Pierre (1969). Late Latin Writers and Their Greek Sources. Trans. H. E. Wedeck. Cambridge: Harvard University Press.

Courcelle, Pierre (1973). "Ambroise de Milan et Calcidius." En Willem den Boer et al. (eds.). Romanitas et Christianitas: Studia Iano Henrino Waszink A. D. Vi Kal. Nou. A. MCMLXXIII XIII lustra complenti oblata. Amsterdam: North-Holland, 45-53.

Cristante, Lucio (1987). Martiani Capellae De nuptiis Philologiae et Mercurii, liber IX. Padova: Editrice Antenore.

Deiters, Hermann (1881). Studien zu den griechischen Musikern. Über das Verhältnis des Martianus Capella zu Aristides Quintilianus. Posen: Merzbach.

Flamant, Jacques (1977). Macrobe et le néo-platonisme latin, à la fin du IVe siècle, Études Préliminaires aux Religions Orientales dans 1'Empire Romain. Leiden: E. J. Brill.

García López, José; Francisco Javier Pérez Cartagena y Pedro Redondo Reyes (2012). La música en la Antigua Grecia. Murcia: Editum.

Garrido Domené, Fuensanta (2012). "Lo que vibra es el yunque: análisis de Nicom. Harm. VI, pp. 245.18-248.26.” $C F C(G)$ 22: 127-140. 
Garrido Domené, Fuensanta (2013). "El universo harmónico platónico (Ti. 35b-36b) según Nicómaco de Gerasa (Harm. VIII; pp. 250.3-252.2 Jan)." Euphrosyne 41: 191-204.

Garrido Domené, Fuensanta (2016). Los teóricos menores de la música griega. Barcelona: Cerix.

Godwin, Joscelyn (1987). Harmonies of Heaven and Earth. Rochester, VT: Inner Traditions International.

Godwin, Joscelyn (1993). The Harmony of the Spheres. Rochester, VT: Inner Traditions International.

Guillaumin, Jean-Baptiste (2003). Martianus Capella, Les noces de Philologie et de Mercure. Livre VII: L'Arithmétique. Paris, Les Belles Lettres.

Hagel, Stefan (2009). Ancient Greek Music: A New Technical History. Cambridge: Cambridge University Press.

Heath, Thomas (1981). A History of Greek Mathematics. Vol. I. New York. Huglo, Michel (1990). "The Study of Ancient Sources of Music Theory in the Medieval Universities." En André Barbera (ed.). Music Theory and Its Sources: Antiquity and the Middle Ages. Notre Dame, IN: University of Notre Dame Press, 150-172.

Hultsch, Fridericus (1867). Censorini de die natali liber. Leipzig: B. G. Teubner.

Jacobsson, Martin (ed.) (2017). Augustinus, De musica. Berlin-Boston: De Gruyter.

Jan, Karl von (1894). "Die Harmonie der Shpären.” Philologus 52: 13-37.

Jan, Karl von (1895), Musici scriptores Graeci (MSG). Aristoteles, Euclides, Nicomachus, Bacchius, Gaudentius, Alypius et melodiarum veterum quidquid exstat. Recognovit prooemiis et indice instruxit Carolus Janus. Annexae sunt tabulae. Leipzig: B. G. Teubner.

Keil, Heinrich (ed.) (1961). Grammatici Latini. Vol. V. Hildesheim: Olms. Lenaz, Luciano (1972). "Marziano Capella." Cultura e scuola 44: 50-59. Luque Moreno, Jesús (2011-2012). "Letras, notas y estrellas.” Revista Internacional de Investigación sobre Magia y Astrología Antiguas 11 (2011): 506-517 y 12 (2012): 133-169.

Luque Moreno, Jesús (2014). Hablar y cantar. La música y el lenguaje (concepciones antiguas). Granada: EUG.

Luque Moreno, Jesús; Antonio, López Eisman (2007). San Agustín. Sobre la música. Seis libros. Madrid: Gredos.

Marrou, Henri-Irénée (1985). Historia de la educación en la Antigüedad. Trad. Y. Barja de Quiriga. Madrid: Akal. 
Mathiesen, Thomas J. (1998). Strunk's Source Readings in Music History, Vol. I. Greek View of Music. New York.

Mathiesen, Thomas J. (2000). Apollo's Lyre. Greek Music and Music

Theory in Antiquity and the Middle Ages. Lincoln and London:

University of Nebraska Press.

Michaelides, Solon (1978). The Music of Ancient Greece. An

Encyclopaedia. London: Faber and Faber.

Navarro Antolín, Fernando (2006). Macrobio. Comentario al "Sueño de Escipión” de Cicerón. Madrid: Gredos.

Navarro Antolín, Fernando (2016). Las nupcias de Filología y Mercurio.

Vol. I: Libros I-II. Introducción, edición crítica, traducción y notas.

Madrid: Alma Mater.

Ortega, Alfonso (1988). Obras completas de San Agustín. Madrid: BAC.

Otaola, Paloma (2005). El De Musica de San Agustín y la tradición pitagórico-platónica. Valladolid: Estudio Agustiniano.

Phillips, Nancy (1990). "Classical and Late Sources for Ninth-Century

Teatrises on Music". En André Barbera (ed.). Music Theory and Its

Sources: Antiquity and the Middle Ages. Notre Dame, Indiana:

University of Notre Dame Press, 100-135.

Prada Dussán, Maximiliano (2014). Filosofía en la música de San Agustín.

Tesis doctoral. Madrid. Universidad Complutense.

Ramelli, Ilaria (2001). Marziano Capella. Le nozze di Filologia e Mercurio. Milano.

Reese, Gustave (1989). La música en la Edad Media. Madrid: Alianza Editorial.

Regali, Mario (1990). Commento al "Somnium Scipioni”, Vol. II. Pisa: Giardini.

Sachs, Curt (1940). The History of Musical Instruments. New York: Dover. Sallmann, Nicolaus (1983). Censorini de die natale liber ad Q. Caerellium. Leipzig: B. G. Teubner.

Scarpa, Luigi (1981). Macrobii Ambrosii Theodosii Commentariorum in Somnium Scipionis libri duo. Padova: Liviana.

Schueller, Herbert M. (1988). The Idea of Music: An Introduction to Musical Aesthetics in Antiquity and the Middle Ages. Kalamazoo: Medieval Institute Publications.

Shanzer, Danuta (1986). A Philosophical and Literary Commentary on Martianus Capella's «De Nuptiis Philologiae et Mercurii» Book I. Berkeley-Los Angeles-London: University of California Press. 
Stahl, William Harris; Johnson, Richard; Burge, Evan Laurie (1971). Martianus Capella and the Seven Liberal Arts. Vol. 1: The Quadrivium of Martianus Capella. Latin Traditions in the Mathematical Sciences, 50 B.C-A.C. 1250, with a Study of the Allegory and the Verbal Disciplines. New York-London: Columbia University Press Stahl, William Harris; Richard Johnson; Evan Laurie Burge (1977). Martianus Capella and the Seven Liberal Arts. Vol. 2: The Marriage of Philology and Mercury. New York-London: Columbia University Press. Theiler, Willy (1933). Porphyrius und Augustin. Halle: Niemeyer. West, Martin L. (1992). Ancient Greek Music. Oxford: Clarendon Press. Wrobel, Johann (1876). Platonis Timaeus interprete Chalcidio cum eiusdem commentario. Leipzig: B. G. Teubner. 